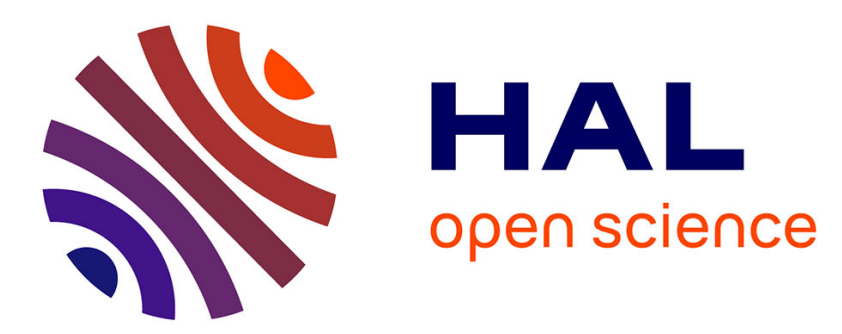

\title{
L'Université numérique francophone mondiale (UNFM) : une expérience pour un enseignement de qualité dans un environnement de pénurie
}

Olivier Archambeau

\section{- To cite this version:}

Olivier Archambeau. L'Université numérique francophone mondiale (UNFM) : une expérience pour un enseignement de qualité dans un environnement de pénurie. Hérodote - Revue de géographie et de géopolitique, 2007, 126, pp.169-173. 10.3917/her.126.0169 . halshs-00731727

\section{HAL Id: halshs-00731727 \\ https://shs.hal.science/halshs-00731727}

Submitted on 26 Apr 2021

HAL is a multi-disciplinary open access archive for the deposit and dissemination of scientific research documents, whether they are published or not. The documents may come from teaching and research institutions in France or abroad, or from public or private research centers.
L'archive ouverte pluridisciplinaire HAL, est destinée au dépôt et à la diffusion de documents scientifiques de niveau recherche, publiés ou non, émanant des établissements d'enseignement et de recherche français ou étrangers, des laboratoires publics ou privés. 


\title{
L'UNIVERSITÉ NUMÉRIQUE FRANCOPHONE MONDIALE (UNFM): UNE EXPÉRIENCE POUR UN ENSEIGNEMENT DE QUALITÉ DANS UN ENVIRONNEMENT DE PÉNURIE
}

\section{Olivier Archambeau}

\author{
La Découverte | « Hérodote »
}

2007/3 n 126 | pages 169 à 173

ISSN 0338-487X

ISBN 9782707152756

Article disponible en ligne à l'adresse :

https://www.cairn.info/revue-herodote-2007-3-page-169.htm

Distribution électronique Cairn.info pour La Découverte.

(C) La Découverte. Tous droits réservés pour tous pays.

La reproduction ou représentation de cet article, notamment par photocopie, n'est autorisée que dans les limites des conditions générales d'utilisation du site ou, le cas échéant, des conditions générales de la licence souscrite par votre établissement. Toute autre reproduction ou représentation, en tout ou partie, sous quelque forme et de quelque manière que ce soit, est interdite sauf accord préalable et écrit de l'éditeur, en dehors des cas prévus par la législation en vigueur en France. Il est précisé que son stockage dans une base de données est également interdit. 


\title{
L’Université numérique francophone mondiale (UNFM) : une expérience pour un enseignement de qualité dans un environnement de pénurie
}

\author{
Olivier Archambeau*
}

La demande en matière d'enseignement sur le continent africain, particulièrement dans les pays les plus déshérités, est immense. Moins peut-être dans l'enseignement supérieur, qui tend à devenir un véritable marché à l'échelle mondiale, notamment pour les élites de ces États, qu'au niveau des formations courtes de santé publique, de la formation des maîtres d'école, de l'apprentissage des savoirs techniques de base ou encore des formations professionnelles continues. Nul n'ignore aujourd'hui la véritable urgence qu'il y a à former une population jeune dans des pays qui manquent cruellement d'infirmiers, de sages-femmes, de techniciens de laboratoire, mais aussi d'instituteurs ou encore de praticiens de l'informatique.

Face à ce constat et sur proposition de Cheick Modibo Diarra, navigateur interplanétaire à la NASA, ambassadeur de bonne volonté de l'UNESCO et ancien dirigeant de l'Université virtuelle africaine, la Fondation Pathfinder basée au Mali et la Fondation pour l'innovation politique ont pris l'initiative du projet UNFM, en janvier 2005.

Il s'agissait de créer une toute nouvelle université qui mettrait à profit les meilleures technologies de l'information et de la communication pour donner au plus grand nombre un accès plus direct à une éducation de qualité, continue et à

* Maître de conférences en géographie à l'université Paris-VIII. 
moindre coût, dans les pays francophones les plus défavorisés d'Afrique d'abord, puis d'Asie. Aider à relever le défi de la formation dans ces pays imposait de proposer de nouvelles formes d'organisation qui allaient naturellement créer de nouveaux liens Nord-Sud, puis très rapidement Sud-Sud.

Structures d'enseignement public souvent en retard, enseignement privé balbutiant se révélant cher et souvent peu fiable, nombre d'élèves et d'étudiants en forte croissance, ressources humaines absentes et besoins cruciaux de formation étaient les principaux problèmes communs rencontrés dans les ministères de l'Éducation nationale et les universités de l'ensemble des pays de l'Afrique subsaharienne. L'UNFM, dès sa création, a donc été pensée et construite en complète collaboration avec les États africains concernés, tant dans les structures que dans les contenus. Restait à construire le projet autour du concept de l'enseignement à distance.

L'idée n'est pas récente en elle-même et, sous une forme ou sous une autre (radio, télévision), l'enseignement à distance a une cinquantaine d'années d'expérience. L'Australie l'utilise dès 1940, le Bangladesh et l'Indonésie depuis 1957, le «Système universitaire de radio et de télévision» apparaît en Chine en 1960 et l'Inde inaugure ses propres systèmes la même année. Aujourd'hui, ce sont plusieurs centaines de milliers d'étudiants dans le monde qui profitent des cours à distance, l'Inde détenant certainement le record d'inscrits avec plus de 34 universités qui proposent ce type d'enseignement.

\section{La synthèse des meilleures technologies au service de l'enseignement}

Aujourd'hui, pour mettre en place un système cohérent d'enseignement à distance, il faut réaliser l'assemblage des meilleures technologies du moment et des meilleures compétences. Si les vecteurs qui permettent la transmission des données audiovisuelles sont aujourd'hui les réseaux Internet sol, les réseaux hertziens et satellitaires, il reste encore à choisir le type de restitution finale des cours. Un problème qui se résume à une simple question: e-learning (travail de l'étudiant sur ordinateur) ou «présence» de l'enseignant sur un écran (télévision ou écran géant), ce qui permet de reconstituer une salle de cours en autant de lieux où il est possible de recevoir le signal enseignant émis en direct.

Le réseau Internet terrestre africain n'étant pas encore, loin s'en faut, suffisant pour accueillir en tous lieux le passage d'un flux capable de transmettre son et vidéo, nous avons donc logiquement choisi la transmission satellite. Pour ce qui est de la restitution des enseignements aux étudiants, c'est la reconstitution de la salle de classe qui a été retenue. L'étudiant suit son cours devant un écran géant (à Bamako ou à Ouagadougou) comme si le professeur était réellement devant lui. 
Un choix évident, dans la mesure où nos étudiants ne sont pas tous capables de se servir seuls d'un ordinateur, même si un technicien en informatique est présent sur les sites et si les machines sont disponibles.

L'architecture de l'ensemble du système, qui comprend les technologies de transmission et la mise en place des sites d'émission et de réception, est le fruit d'une collaboration entre les deux fondations et les partenaires très actifs qui nous ont rejoints dans ce projet : l'HEGP (Hôpital européen Georges-Pompidou), le CNES (Centre national d'études spatiales), Alcatel Space, les universités de Ouagadougou et de Bordeaux, l'École nationale des ingénieurs de Bamako et le Fonds de solidarité numérique (FSN).

La mise au point de ce «kit technique» destiné à équiper rapidement chaque nouveau site de notre Université a été pensée dès l'origine pour être « réplicable » à volonté. Sur le terrain, chaque centre est dirigé par un administrateur aidé par un technicien. Des tuteurs travaillent également avec les étudiants pour le suivi pédagogique. Ils seront bien sûr différents en fonction des formations.

\section{De la phase pilote au modèle de développement}

L'expérience a débuté effectivement en juin 2005 à l'université de Ouagadougou et à l'École nationale des ingénieurs de Bamako. Les premières "formations santé » dispensées, conformément aux demandes des pays concernés, ont été élaborées par l'équipe du professeur Michel Le Bras. Cette première équipe est composée de professeurs français, burkinabés et canadiens. Les formations comprennent trois modules de 120 heures: décision de soins et d'alerte, vaccinologie pratique, VIH/sida, prévention de la transmission de la mère à l'enfant, répartis entre 90 heures de cours interactifs et 30 heures de travaux dirigés. Les 50 étudiants de chaque site ont été sélectionnés selon des critères mis en place par les services des ministères locaux. La plupart d'entre eux travaillaient déjà sur le terrain et venaient avec le désir d'acquérir une formation théorique de base. $90 \%$ d'entre eux ne savaient pas se servir d'un ordinateur.

Les cours dispensés à partir de Paris, à raison de trois heures par jour, sont donc émis vers l'Afrique. Les cours s'arrêtent toutes les vingt minutes pour permettre un dialogue en direct entre les étudiants et le professeur. Dans la salle de cours, les questions, souvent communes aux deux sites, sont rassemblées et envoyées par les tuteurs. À la suite des enseignements, les tuteurs peuvent fournir aux étudiants les explications nécessaires et les aider dans la réalisation de leurs travaux pratiques. Hors des horaires de cours, les ordinateurs restent à la disposition des étudiants de l'UNFM. Ils peuvent se former et surtout, s'ils le désirent, visionner l'ensemble des enseignements qui sont systématiquement enregistrés sur 
DVD et réunis dans une vidéothèque. Fin janvier 2006, les premiers contrôles des connaissances et des enseignements ont été effectués. La première promotion des étudiants de l'UNFM recevra bientôt une «Capacité en décision de santé ».

L'UNFM associe au projet un nombre croissant d'universités francophones du Sud et du Nord. Dans les semaines qui viennent, les sites de Niamey (Niger) et de Brazzaville (République démocratique du Congo) seront à leur tour équipés et, avant la fin de l'année, deux autres pays de l'Afrique de l'Ouest suivront. Si les enseignements en matière de santé restent prioritaires, la formation des maîtres, l'apprentissage de l'informatique et de la gestion compléteront très bientôt l'offre UNFM, créant ainsi une sorte de «hub» francophone pour l'éducation de base. La participation et les efforts des pays partenaires qui ont accueilli ce projet avec enthousiasme, le financement des différents programmes et l'accroissement du nombre d'étudiants vont rapidement et sensiblement réduire le coût des formations par étudiant, l'objectif étant de descendre en deçà de 100 dollars en moins de trois ans.

En adoptant les Objectifs du millénaire pour le développement (ODM) lors du sommet de New York, en août 2000, qui visent notamment à assurer d'ici 2015 l'éducation primaire pour tous, à promouvoir l'autonomisation des femmes, à réduire de deux tiers la mortalité infantile, à améliorer la santé maternelle (réduire la mortalité de trois quarts), à combattre le VIH/sida, le paludisme et la tuberculose, ou encore à réduire l'extrême pauvreté et la faim, la communauté internationale a lancé un appel aux États et aux bonnes volontés du monde entier. L'introduction de technologies de communication performantes peut permettre un véritable saut qualitatif et quantitatif dans l'aide au développement des pays les plus pauvres. Bien utilisées, ces technologies permettront même aux jeunes générations d'opérer un véritable rattrapage du savoir commun mondial.

La grande majorité des chefs de gouvernement africains ne s'y sont pas trompés. Leur présence massive au Sommet mondial sur la société de l'information (SMSI) à Tunis, en novembre 2005, a montré tout l'intérêt qu'ils portent à ces nouvelles voies possibles pour le développement, à ces innovations qui deviennent politiques. De nouvelles pratiques liées à ces technologies prennent maintenant leur place dans les plans de développement nationaux.

En parallèle à cette évolution, l'apparition et la mise en place de nouveaux modes de contributions supranationales, destinées à résoudre les problèmes globaux - taxe sur les billets d'avion, proposée par le président français Jacques Chirac, ou le «100\% numérique» voulu par le Fonds de solidarité numérique (FSN) -, vont dans le bon sens. Ces financements vont aider à développer de nouvelles formes d'innovations pragmatiques qui mêlent sphère étatique et société civile. C'est en prenant en compte ce nouvel environnement, avec la plus grande modestie mais aussi le fort désir de réussir, que l'Université numérique franco- 
phone mondiale, sous la direction de Cheick Modibo Diarra et du professeur JeanDidier Vincent, s'est fixé pour objectif de contribuer à la réduction des inégalités, par la mise à disposition pour le plus grand nombre d'une éducation de qualité.

\section{Bibliographie}

ARCHAMBEAU Olivier, «L'Université numérique francophone mondiale. Une expérience pour un enseignement de qualité dans un environnement de pénurie», Point de vue, $\mathrm{n}^{\circ} 12,10$ février 2006. 